\title{
Modelling of the Hand-Foot-Mouth-Disease with the Carrier Population
}

\author{
Ruzhang Zhao, Lijun Yang* \\ Department of Mathematical Science, Tsinghua University, China. \\ * Corresponding author. Email: lyang@math.tsinghua.edu.cn, zrz15@mails.tsinghua.edu.cn \\ Manuscript submitted January 10, 2018; accepted March 8, 2018. \\ doi: 10.17706/ijapm.2019.9.1.54-64
}

\begin{abstract}
This paper focuses on the modelling of the hand, foot and mouth disease (HFMD) with people carrying the pathogen. HFMD has become widespread in China, and the Chinese public health has been seriously threatened. The modelling of the HFMD has already taken several aspects into consideration but in the traditional modelling of infectious disease, the people who are not infected but carry the pathogen are ignored. In this paper, the "carrier" is included in the modelling of the HFMD and the newly developed model is proved to play a key role in the modelling and stability analysis of the HFMD.
\end{abstract}

Key words: HFMD, carrier population, SIRC model, stability analysis.

\section{Introduction}

The existing solutions to methods for the development of infection models have been widely taken into consideration for a long time. Additionally, the modeling to control the spread of some infectious disease is also widely used, and the theoretical basis has been studied, [1]-[4]. Different kinds of infectious diseases will share many important characteristics, such as whether the people who recover from them will become immune to them. However, the specific characteristic of some infectious diseases will be largely different for their spread, [5]. The previous models for the spread of infectious diseases do not really work for the hand, foot and mouth disease (HFMD), due to its specific characteristic, [6]

The most significant feature of the HFMD is that people older than 10 years of age have lifelong immunization and its pathogen can be spread via interpersonal communication (Centers for Disease Control and Prevention, 2017). If we ignore this feature and simply apply the existing models to the spread of the HFMD, the criterion for the existence of the disease-free equilibrium may be satisfied. Many mathematical models have been constructed and proved reasonable to explain the spread of HFMD, [7]-[11]. However, if we apply the SIRC model (Susceptible, Infected, Removed and Carrier model) to the modelling process, we may obtain a result that is contradictory to the previous result, which can be clearly seen in the following simulation section. The result appeals to us because if policies are made under the previous result, the existence of the endemic equilibrium is ignored and the control of the HFMD will be lower than required, which is dangerous for the public. The material already present will bring the problem into the disease transmission coefficient at a given time, [9], [12], [13]. However, the change of the disease transmission coefficient is not easy to evaluate. Now, we can provide another solution to the problem. We can extend the models further by including the carrier population, people who are not infected but carry the pathogen, in the modelling process, which has never been tried in previous modelling studies. The new model is called the 
model is called the SIRC model, which is the main topic of this paper.

The HFMD is closely connected with the kind of people mentioned above, because the adults carrying the pathogen will be an important reason for children catching HFMD. That makes the model described in this paper reasonable.

The rest of the paper consists of three sections after the development of the HFMD model including the carrier population. In section 2, we discuss the disease-free equilibrium. Section 3 provides the discussion on endemic equilibrium. Section 4 comprises the overall discussion of the paper and section 5 provides some simulations using the SIRC model.

\section{Assumptions and Notations of the Modelling}

This section gives the assumptions made in the modelling and lists the basic notations used in the paper.

\subsection{Assumptions of the Model}

In the modelling, the following assumptions are always made:

The population is divided into four groups: susceptible (S), infected (I), removed (R) and carrier (C). The removed population group comprises the people immune to the HFMD and the carrier population group comprises the people carrying the pathogen but who are not infected. The total number of people is denoted as $\mathrm{N}$, which equals to the sum of people included in the $\mathrm{S}, \mathrm{I}, \mathrm{R}$ and $\mathrm{C}$ population groups. The variables change with time, which means that they are a function of time.

It is assumed that the individuals who are older than 5 years of age have lifelong immunization, because the HFMD is a disease caused by a group of viruses that mainly infect people younger than 5 years of age. And it is assumed that all the new born children are susceptible to HFMD.

It is assumed that the people who recover from the HFMD have lifelong immunization.

The population is growing at a low speed, which means that the natural birth rate is larger than the natural mortality rate and we do not consider that the people move in and out the city. Additionally, it is assumed that the natural birth rate is larger than the mortality rate due to HFMD.

The standard incidence ratio is used.

\subsection{Notations of the Paper}

In this paper, the following notations are used.

Susceptible, S; infected, I; removed, R; carrier, C; total number of people, N; ratio of S to N, s; ratio of I to N, $\mathrm{i}$; ratio of $\mathrm{R}$ to $\mathrm{N}$, r; ratio of $\mathrm{C}$ to $\mathrm{N}$, c.

Natural birth rate, b; natural mortality rate, $d$; contact coefficient between the susceptible and infected population, ${ }^{\beta}$; contact coefficient between susceptible population and carrier population, $\beta_{1}$; ratio of people who get lifelong immunization at an age above 10 years old (Centers for Disease Control and Prevention, 2017), $\sigma$; mortality rate due to illness, $\alpha$; recovery coefficient of the infected people, $\gamma$; transferred coefficient from the susceptible population to the carrier population, ${ }^{\zeta}$; exclusive coefficient of the carrier population, $\rho$.

The standard incidence between the susceptible and infected population, $\beta S I / N$; the standard incidence between the susceptible and infected population, $\beta_{1} S C / N$.

\section{Modelling of the HFMD with the Carriers}

The following flow chart illustrates the propagation of HFMD as obtained by the model. where $b N$ denotes the number of new-borns and $d S$ is the number of new-borns deaths due to natural 


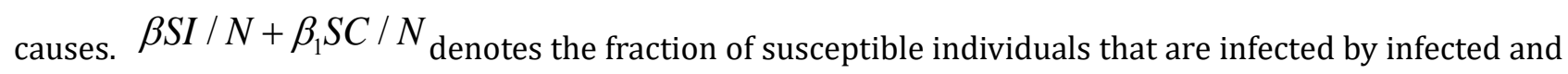
carrier individuals. $\sigma S$ and $\zeta S I / N$ denote the number of individuals who are removed and the fraction of carrier individuals respectively. $(d+\alpha) I$ denotes the infected population who are killed by

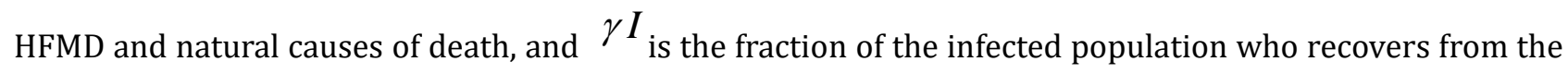
disease. $d R$ and $d C$ also denote the number of natural deaths of the removed individuals and carrier individuals respectively. $\zeta R I / N$ is the fraction of the removed population who become carrier individuals and $\rho C$ is the fraction of the carrier individuals who are moved into ' $R$ ' population group.

From the flowchart depicted above, the following system of differential equations can be derived.

$$
\left\{\begin{array}{l}
\frac{d S}{d t}=b N-(d+\sigma) S-(\beta+\zeta) \frac{S I}{N}-\beta_{1} \frac{S C}{N} \\
\frac{d I}{d t}=\beta \frac{S I}{N}+\beta_{1} \frac{S C}{N}-(d+\alpha+\gamma) I \\
\frac{d R}{d t}=\sigma S+\gamma I+\rho C-d R-\zeta \frac{R I}{N} \\
\frac{d C}{d t}=\zeta \frac{R I}{N}+\zeta \frac{S I}{N}-(\rho+d) C
\end{array}\right.
$$

The sum of the four differential equations is $d N / d t=(b-d) N-\alpha I$. Using the transformation $(S, I, R, C)=N(s, i, r, c)$, the following system of differential equations, which has been normalized, can be obtained.

$$
\left\{\begin{array}{l}
\frac{d s}{d t}=b-(b+\sigma) s-(\beta+\zeta-\alpha) s i-\beta_{1} s c \\
\frac{d i}{d t}=\beta s i+\beta_{1} s c-(b+\alpha+\gamma) i+\alpha i^{2} \\
\frac{d r}{d t}=\sigma s+\gamma i+\rho c-b r+(\alpha-\zeta) r i \\
\frac{d c}{d t}=\zeta r i+\zeta s i-(\rho+b) c+\alpha c i \\
1=s+i+r+c
\end{array}\right.
$$

Then, eliminating the variable $r$, the system with three variables is obtained.

$$
\left\{\begin{array}{l}
\frac{d s}{d t}=b-(b+\sigma) s-(\beta+\zeta-\alpha) s i-\beta_{1} s c \\
\frac{d i}{d t}=\beta s i+\beta_{1} s c-(b+\alpha+\gamma) i+\alpha i^{2} \\
\frac{d c}{d t}=\zeta i-\zeta i^{2}-(\rho+b) c+(\alpha-\zeta) c i
\end{array}\right.
$$




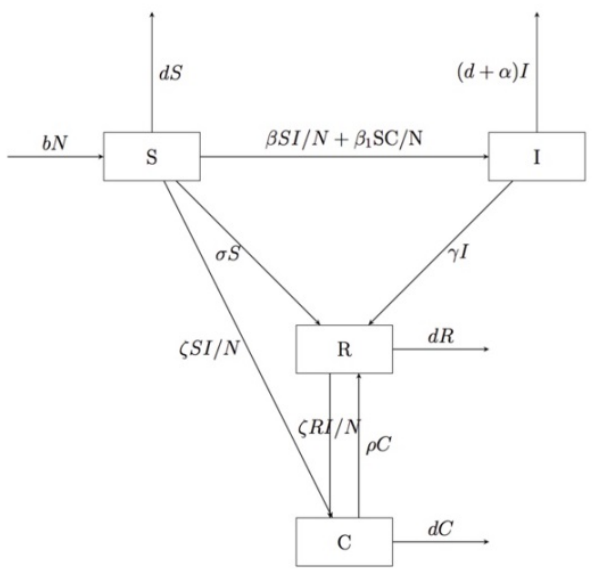

Fig. 1. Modelling of the HFMD with the carrier population.

\section{Basic Reproduction Number}

The basic reproduction number (BRN) in the normalized system needs to be modified. When there are only susceptible individuals in the system, the sum of the ratio of the individuals infected by one infected individual to the total population and the ratio of the individuals infected by one carrier individual to the total population is what we redefined as the BRN.

Letting the right-hand side of the system (3) be equal to zero, we obtain the disease-free equilibrium $U_{0}(b /(b+\sigma), 0,0)$, which means that when there are only susceptible individuals and the ratio of them is $b /(b+\sigma)$, the system is in equilibrium.

When only the recovery is considered, the average morbidity cycle is $1 / \gamma$, and when the natural mortality and the mortality due to illness are both added, the average morbidity period is $1 /(b+\alpha+\gamma)$

Likewise, the average carrying period of the carrier individuals is $1 /(\rho+b)$.

According the definition of the BRN, we assume that there are only susceptible individuals. The individuals infected by one infected person is $\beta s_{0} /(b+\alpha+\gamma)$. The carrier individual is equal to $\zeta$, so the individuals infected by the carrier individual is $\zeta \beta_{1} s_{0} /(\rho+b)(b+\alpha+\gamma)$.

Thus, BRN is equal to the sum of the two kinds of individuals.

$$
R_{0}=\frac{\beta s_{0}}{(b+\alpha+\gamma)}+\frac{\zeta \beta_{1} s_{0}}{(\rho+b)(b+\alpha+\gamma)}=\frac{b \beta}{(b+\sigma)(b+\alpha+\gamma)}+\frac{b \zeta \beta_{1}}{(b+\sigma)(\rho+b)(b+\alpha+\gamma)}
$$

\section{Disease-Free Equilibrium}

The disease-free equilibrium $U_{0}(b /(b+\sigma), 0,0)$ was obtained above, and the local stability and global asymptotic stability of the disease-free equilibrium will be discussed next.

\subsection{Local Stability of Disease-Free Equilibrium}

Theorem 4.1: When $R_{0}<1$, the disease-free equilibrium is locally asymptotically stable.

When $R_{0}>1$, the disease-free equilibrium is unstable. 
Proof: The definition domain is $\{(s, i, c) \mid 0 \leq s \leq 1,0 \leq i \leq 1,0 \leq c \leq 1\}$.

Denote the disease-free equilibrium as $\left(s_{0}, i_{0}, c_{0}\right)$, then the following Jacobian matrix can be obtained.

$$
\left(\begin{array}{ccc}
-(b+\sigma) & -(\beta+\zeta-\alpha) s_{0} & -\beta_{1} s_{0} \\
0 & \beta s_{0}-(b+\alpha+\gamma) & \beta_{1} s_{0} \\
0 & \zeta & -(\rho+b)
\end{array}\right)
$$

Let us denote three eigenvalues as $\xi_{1}, \xi_{2}$ and $\xi_{3}$. It is then easy to get the three following equations.

$$
\left\{\begin{array}{l}
\xi_{1}=-(b+\sigma) \\
\xi_{2}+\xi_{3}=\beta s_{0}-(b+\alpha+\gamma)-(\rho+b) \\
\xi_{2} \xi_{3}=\left[-\beta s_{0}+(b+\alpha+\gamma)\right](\rho+b)-\zeta \beta_{1} s_{0}=(b+\alpha+\gamma)(\rho+b)\left(1-R_{0}\right)
\end{array}\right.
$$

Using the condition " $R_{0}<1$ ", we can get $\xi_{1}<0, \xi_{2}+\xi_{3}<0, \xi_{2} \xi_{3}>0$, which can also be transformed into $\xi_{1}<0, \xi_{2}<0$ and $\xi_{3}<0$. Thus, the disease-free equilibrium $U_{0}$ is locally asymptotically stable.

Using the condition " $R_{0}>1$ ", we can get $\xi_{2} \xi_{3}<0$, where there must be one eigenvalue larger than zero. Thus, the disease-free equilibrium $U_{0}$ is unstable.

\subsection{Global Asymptotic Stability of Disease-Free Equilibrium}

Theorem 4.2: $\widehat{R_{0}}=\frac{\beta}{(b+\alpha+\gamma)}+\frac{\zeta \beta_{1}}{(\rho+b)(b+\alpha+\gamma)}$. When $\widehat{R_{0}} \leq 1$, the disease-free equilibrium $U_{0}$ is globally asymptotically stable.

Proof: To narrow the definition domain of "s", the following differential inequality will be applied.

$$
\frac{d s}{d t}=b-(b+\sigma) s-(\beta+\zeta-\alpha) s i-\beta_{1} s c \leq b-(b+\sigma) s
$$

The domain of " $\mathrm{s}$ " can be easily obtained $-s_{0} \leq\left(s-s_{0}\right) \leq 0$.

Using the method of undetermined coefficients, the Liapunov function $L$ can be constructed.

$$
\begin{gathered}
L=k_{1} i+k_{2} c \\
\frac{d L}{d t}=k_{1} \frac{d i}{d t}+k_{2} \frac{d c}{d t}=k_{1}\left[\beta s i+\beta_{1}\left(s-s_{0}\right) c+\beta_{1} s_{0} c-(b+\alpha+\gamma) i+\alpha i^{2}\right] \\
+k_{2}\left[\zeta i-\zeta i^{2}-(\rho+b) c+(\alpha-\zeta) c i\right] \\
\frac{d L}{d t}=k_{1} \beta_{1}\left(s-s_{0}\right) i+c\left[k_{1} \beta_{1} s_{0}-k_{2}(\rho+b)\right]+ \\
i\left[k_{1} \beta s+\left(k_{1} \alpha-k_{2} \zeta\right) i+k_{2}(\alpha-\zeta) c+k_{2} \zeta-k_{1}(b+\alpha+\gamma)\right]
\end{gathered}
$$


Let the coefficient at the front of "c" be equal to zero. We can then get $k_{1} \beta_{1} s_{0}-k_{2}(\rho+b)=0$.

We can also assume that $k_{1}=1, k_{2}=\beta_{1} s_{0} /(\rho+b)$. Also, using the condition $s+i+c \leq 1$, $\frac{d L}{d t} \leq i\left[\beta s+\left(\alpha-k_{2} \zeta\right) i+k_{2}(\alpha-\zeta) c+k_{2} \zeta-(b+\alpha+\gamma)\right]$ can be obtained.

$$
\begin{array}{r}
\quad \leq i\left[\max \left\{\beta,\left(\alpha-k_{2} \zeta\right), k_{2}(\alpha-\zeta)\right\}+k_{2} \zeta-(b+\alpha+\gamma)\right] \\
\hat{R}_{0}=\frac{\beta}{(b+\alpha+\gamma)}+\frac{\zeta \beta_{1}}{(\rho+b)(b+\alpha+\gamma)} \leq 1, \text { we can get } d L / d t \leq 0 .
\end{array}
$$

Then, let $d L / d t=0$, the solution to the equation is $\{i \mid i=0\}$.

In conclusion, $L \geq 0, L^{\prime} \leq 0$ and $L^{\prime}=0$ if $\mathrm{f} i=0$. Also, $\{i \mid i=0\}$ is the global attractive set under the condition $\widehat{R_{0}} \leq 1$.

Thus, the theorem has been proofed.

\section{Endemic Equilibrium}

Before the analysis, we need to make the following additional assumptions. $\alpha<\zeta, \sigma$ and $\zeta \beta_{1}<(\zeta-\alpha) \beta$

\subsection{Existence and Uniqueness of Endemic Equilibrium}

Theorem 5.1: When $R_{0}>1$, the endemic equilibrium exists and is unique.

Proof: Let us denote the endemic equilibrium as $U^{*}\left(s^{*}, i^{*}, c^{*}\right)$ and $s^{*}, i^{*}, c^{*}$ can be solved with the following equations.

$$
\left\{\begin{array}{l}
b-(b+\sigma) s^{*}-(\beta+\zeta-\alpha) s^{*} i^{*}-\beta_{1} s^{*} c^{*}=0 \\
\beta s^{*} i^{*}+\beta_{1} s^{*} c^{*}-(b+\alpha+\gamma) i^{*}+\alpha\left(i^{*}\right)^{2}=0 \\
\zeta i^{*}-\zeta\left(i^{*}\right)^{2}-(\rho+b) c^{*}+(\alpha-\zeta) c^{*} i^{*}=0
\end{array}\right.
$$

The feasible region of the equations is $\Omega=\left\{\left(s^{*}, i^{*}, c^{*}\right) \mid 0 \leq s^{*}, i^{*}, c^{*} \leq 1\right\}$.

And applying $i^{*}$ to express $s^{*}$ and $c^{*}$, the following can be obtained

$$
s^{*}=\frac{b-(b+\alpha+\gamma) i^{*}+\alpha\left(i^{*}\right)^{2}}{(b+\sigma)-(\alpha-\zeta) i^{*}}, c^{*}=\frac{\zeta i^{*}-\zeta\left(i^{*}\right)^{2}}{(\rho+b)-(\alpha-\zeta) i^{*}} .
$$

And $i^{*}$ satisfies the following equation.

$$
\begin{aligned}
& {\left[(\rho+b)-(\alpha-\zeta) i^{*}\right] b\left[(b+\sigma)-(\alpha-\zeta) i^{*}\right]-\left[(\rho+b)-(\alpha-\zeta) i^{*}\right](b+\sigma)\left[b-(b+\alpha+\gamma) i^{*}+\alpha\left(i^{*}\right)^{2}\right]-} \\
& {\left[(\rho+b)-(\alpha-\zeta) i^{*}\right](\beta+\zeta-\alpha)\left[b-(b+\alpha+\gamma) i^{*}+\alpha\left(i^{*}\right)^{2}\right] i^{*}-\beta_{1}\left[b-(b+\alpha+\gamma) i^{*}+\alpha\left(i^{*}\right)^{2}\right]\left(\zeta i^{*}-\zeta\left(i^{*}\right)^{2}\right)}
\end{aligned}
$$


Denoting equation (14) as $W\left(i^{*}\right)$ and analyzing the existence of $i^{*}$ in the endemic equilibrium.

$$
\begin{aligned}
& W\left(i^{*}\right) \text { can be written as } W\left(i^{*}\right)=g_{1}\left(i^{*}\right)^{4}+g_{2}\left(i^{*}\right)^{3}+g_{3}\left(i^{*}\right)^{2}+g_{4}\left(i^{*}\right) . \\
& g_{1}=\zeta \alpha \beta_{1}+(\alpha-\zeta)(\beta+\zeta-\alpha) \alpha \\
& g_{4}=-(\rho+b) b(\alpha-\zeta)+(\rho+b)(b+\sigma)(b+\alpha+\gamma)-(\rho+b)(\beta+\zeta-\alpha) b-\beta_{1} b \zeta
\end{aligned}
$$

Then,

$$
\begin{gathered}
W(0)=0, W(1)=(\rho+b-\alpha+\zeta)[b(b+\sigma-\alpha+\zeta)+\gamma(b+\sigma+\beta+\zeta-\alpha)]>0, \\
W^{\prime}(0)=g_{4}=-(\rho+b) b(\alpha-\zeta)+(\rho+b)(b+\sigma)(b+\alpha+\gamma)-(\rho+b)(\beta+\zeta-\alpha) b-\beta_{1} b \zeta \\
=(\rho+b)(b+\sigma)(b+\alpha+\gamma)\left(1-R_{0}\right)<0
\end{gathered}
$$

Thus, there exists $i^{*}$ in the equation $W\left(i^{*}\right)=0$.

Regarding the uniqueness of $i^{*}$, let us perform the following analysis.

$$
g_{1}=\alpha\left[\zeta \beta_{1}-(\zeta-\alpha) \beta-(\zeta-\alpha)^{2}\right]<0
$$

Accordingly, the cubic function $W^{\prime}\left(i^{*}\right)$ has a negative coefficient of cubic term. And, as $W^{\prime}(0)<0$, we can obtain the image of $W^{\prime}\left(i^{*}\right)$ and the y-axis intersect, at most, at two points, which means that the function $W^{\prime}\left(i^{*}\right)$ has, at most, two extreme points. And as $W(0)=0, W(1)>0, W^{\prime}(0)<0$, the image of $W^{\prime}\left(i^{*}\right)$ and the y-axis intersect at only one point, which means that the equation $W\left(i^{*}\right)=0$ only has one solution at the feasible region of $i^{*}$.

Meanwhile, it is easy to get $s^{*}>0, c^{*}>0, s^{*} \leq \frac{b+\alpha}{b+\sigma} \leq 1, c^{*} \leq \frac{\zeta i^{*}}{\zeta i^{*}+(\rho+b-\alpha)} \leq 1$. And since $i^{*}$ is unique, $s^{*}$ and $c^{*}$ are also unique.

In conclusion, the endemic equilibrium $U^{*}\left(s^{*}, i^{*}, c^{*}\right)$ existence has been proved and it is unique.

\subsection{Local Stability of Endemic Equilibrium}

Theorem 5.2: When $R_{0}>1$ and $R_{1}=\frac{b \beta_{1} \zeta}{(\rho+b)(b+\alpha+\gamma)(b+\sigma)}<1$, the endemic equilibrium is locally asymptotically stable.

Proof: Let $y=(\hat{s}, \hat{i}, \hat{c})=\left(s-s_{0}, i, c\right)$, and calculate linear approximation system $y^{\prime}=A y$, where A is:

$$
\left(\begin{array}{ccc}
-(b+\sigma)-(\beta+\zeta-\alpha) i^{*}-\beta_{1} c^{*} & -(\beta+\zeta-\alpha) s^{*} & -\beta_{1} s^{*} \\
\beta i^{*}+\beta_{1} c^{*} & \beta s^{*}-(b+\alpha+\gamma)+2 \alpha i^{*} & \beta_{1}{ }^{*} \\
0 & \zeta-2 \zeta i^{*}+(\alpha-\zeta) c^{*} & -(\rho+b)+(\alpha-\zeta) i^{*}
\end{array}\right)
$$


Applying the Routh-Hurwitz criterion to prove the local stability of endemic equilibrium. Where the Routh-Hurwitz criterion states that if all the order principal minor determinants are more than zero, the matrix is locally asymptotically stable. Denoting $\Delta_{i}$ as the $i$ th order principal minor determinant. As for A, where

$$
\begin{gathered}
\Delta_{1}=-(b+\sigma)-(\beta+\zeta-\alpha) i^{*}-\beta_{1} c^{*}<0 \\
\Delta_{2}=\left[(b+\sigma)+(\beta+\zeta-\alpha) i^{*}+\beta_{1} c^{*}\right]\left(-\beta s^{*}+(b+\alpha+\gamma)-2 \alpha i^{*}\right)+(\beta+\zeta-\alpha) s^{*}\left(\beta i^{*}+\beta_{1} c^{*}\right) \\
=\left[(b+\sigma)+(\beta+\zeta-\alpha) i^{*}+\beta_{1} c^{*}\right]\left((b+\alpha+\gamma)-2 \alpha i^{*}\right)-(b+\sigma) \beta s^{*}+(\zeta-\alpha) \beta_{1} s^{*} c^{*} \\
>\frac{1}{i^{*}}\left[(b+\sigma)+(\beta+\zeta-\alpha) i^{*}+\beta_{1} c^{*}\right]\left(\beta_{1} s^{*} c^{*}-\alpha\left(i^{*}\right)^{2}\right) \\
=\left[(b+\sigma)+(\beta+\zeta-\alpha) i^{*}+\beta_{1} c^{*}\right]\left[(b+\alpha+\gamma)-\beta s^{*}-2 \alpha i^{*}\right]>0 \\
\Delta_{3}=\left[-(\rho+b)+(\alpha-\zeta) i^{*}\right] \Delta_{2}+\left(\zeta-2 \zeta i^{*}+(\alpha-\zeta) c^{*}\right) \\
\left\{\left[(b+\sigma)+(\beta+\zeta-\alpha) i^{*}+\beta_{1} c^{*}\right] \beta_{1} s^{*}-\beta_{1} s^{*}\left(\beta i^{*}+\beta_{1} c^{*}\right)\right\}
\end{gathered}
$$

When $\left(\zeta-2 \zeta i^{*}+(\alpha-\zeta) c^{*}\right) \leq 0$, it is easy to get $\Delta_{3}<0$.

When $\left(\zeta-2 \zeta i^{*}+(\alpha-\zeta) c^{*}\right)>0$, the following transformation can be obtained.

$$
\begin{gathered}
\Delta_{3}=\left[(b+\sigma)+(\beta+\zeta-\alpha) i^{*}\right]\left\{\left[-(\rho+b)+(\alpha-\zeta) i^{*}\right]\right. \\
\left.\left((b+\alpha+\gamma)-2 \alpha i^{*}\right)+\left(\zeta-2 \zeta i^{*}+(\alpha-\zeta) c^{*}\right) \beta_{1} s^{*}\right\} \\
+\left[(\rho+b)+(\zeta-\alpha) i^{*}\right](b+\sigma) \beta s^{*}+\left[-(\rho+b)+(\alpha-\zeta) i^{*}\right] \\
\beta_{1} c^{*}\left[(b+\alpha+\gamma)-2 \alpha i^{*}+(\zeta-\alpha) s^{*}\right]-\left(\zeta-2 \zeta i^{*}+(\alpha-\zeta) c^{*}\right) \beta_{1} s^{*} \beta i^{*}
\end{gathered}
$$

According to the assumption,

$$
(b+\alpha+\gamma)-2 \alpha i^{*}+(\zeta-\alpha) s^{*}>0 .
$$

Thus, only the following inequality (27) needs to be proven in order to prove $\Delta_{3}<0$.

$$
\begin{gathered}
{\left[-(\rho+b)+(\alpha-\zeta) i^{*}\right]\left((b+\alpha+\gamma)-2 \alpha i^{*}-\beta s^{*}\right)\left[(b+\sigma)-(\alpha-\zeta) i^{*}\right]} \\
+\left(\zeta-2 \zeta i^{*}+(\alpha-\zeta) c^{*}\right) \beta_{1}\left[b-(b+\alpha+\gamma) i^{*}+\alpha\left(i^{*}\right)^{2}\right]<0
\end{gathered}
$$

Applying the condition $R_{1}<1$, the inequality (27) is true.

Thus, $\Delta_{3}<0$.

In conclusion, according to the Routh-Hurwitz criterion, the endemic equilibrium is locally asymptotically stable.

\section{Discussion}

Considering the problem that is caused by the specific characteristic of the HFMD, the existing models for describing the spread of infectious diseases are not suitable for the HFMD. The aim of this study was to provide a solution to this problem. The existing studies focus on changing the existing models and produce results that are not easy to analyze and cannot reflect the characteristics of the spread of the HFMD. 
The most significant factors for the HFMD cannot be adequately described by existing models. The reason for the development of the model is to provide a more suitable model for the spread of the HFMD.

The most innovative part for the SIRC model with carriers is the introduction of the new individuals, which is also a novel approach to improve the previous models according to the specific characteristics of the HFMD. In the previous models, the spread process of the infectious model includes many parts, such as different environment for spread, the activities among people, etc. Regarding the HFMD, it is a disease that is spread from person to person through coughing and sneezing, or contact with an infected person (Centers for Disease Control and Prevention, 2017). For example, if a kid who has no outdoors activities suffers from the HFMD, the kid's parents may be the reason for his infection with the virus that causes this disease. From a pathological perspective, the people around the kids play an important part in the spread of the disease because the pathogens of the HFMD, such as the Coxsackie virus, are highly contagious (Graham, 2012), thus it is easy to spread by the individuals that carry these viruses. If we ignore this fact, the spread model will not adequately describe the real situation of the HFMD and can even be wrong.

Once we include the carrier individuals in the modelling, the spread part can be analyzed instead of being regarded as a normal part of the spread process. The interrelationships between the carrier individuals and other individuals are analogous to those with the existing individuals. Once the existence and characteristics of the disease-free equilibrium and the endemic equilibrium are proved, the SIRC model can been proved to be rational. Such work was completed in section 5 and section 6 . The advantages of the SIRC model over the evaluation of the change of the coefficients will be clear, because, instead of ignoring carrier individuals, we will not only include the them in the spread system but we also will find an easier way to determine the criterion for the disease. Making the carrier an individual instead of combining all the spread media together can benefit the analysis of the influence of the carrier individuals.

For the additional application of the SIRC model, as can be seen from the modelling process, the most important consideration of the SIRC model is the carrier individual. Many similar infectious diseases share the same characteristics, for example, varicella. Thus, if the SIRC model can also be applied to the modelling of such diseases, the spread part will be more clearly analyzed. Moreover, the criterion for the existence of disease-free equilibrium and endemic equilibrium are easy to operate in ways that are suitable for the medical evaluation.

In the following simulation section, we can obtain different results with the SIR model and SIRC model, for the existing of carriers.

Encouraged by the consideration of this study, we can also change the spread of some infectious diseases by introducing a new group of individuals, which is a novel idea for analyzing the influence of some parts.

In conclusion, in this paper, we modified the spread process of the HFMD by introducing a new carrier individual group. Additionally, the rationality of the model was proved and the different results obtained compared with the previous models can be used to generate different policies for disease control.

\section{Simulations}

To simulate the prevalence of HFMD under different conditions, let us set two groups of parameters and represent the situations with the following figures.

\subsection{Parameters Settings with Disease-Free Equilibrium}

We may set the parameters as shown in Table 1 . Also, $R_{0}=0.1626<1$ and $\widehat{R_{0}}=0.9882<1$. According to Theorem 5.2, the system has a disease-free equilibrium with global asymptotic stability.

Let us set the initial conditions as $\left(s_{0}, i_{0}, c_{0}\right)=(0.75,0.1,0.15)$ and the results presented in Fig. 2 are obtained. 
Table 1. Parameter Settings

\begin{tabular}{llllllllr}
\hline \hline Parameters & $\mathrm{b}$ & $\beta$ & $\beta_{1}$ & $\sigma$ & $\alpha$ & $\gamma$ & $\zeta$ & $\rho$ \\
\hline Number & 0.013 & 0.11 & 0.05 & 0.066 & 0.003 & 0.117 & 0.02 & 0.1 \\
\hline \hline
\end{tabular}

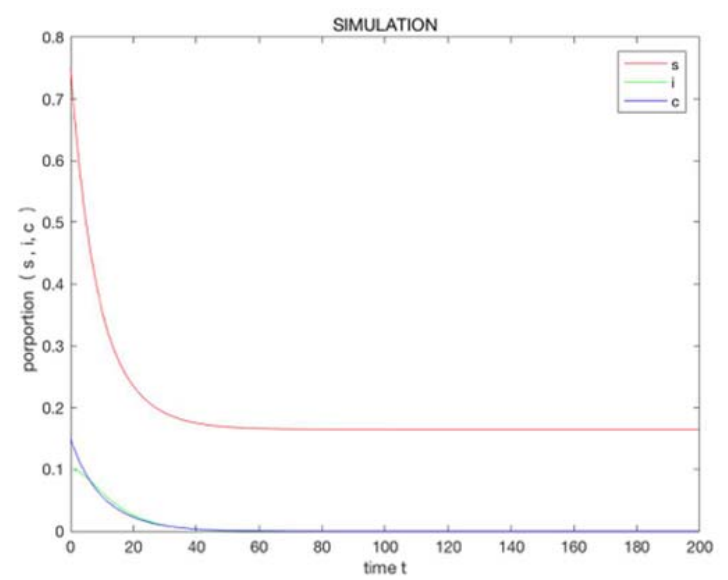

Fig. 2. Simulation with disease-free equilibrium.

The results shown in Fig. 2 reveal that the proportion of three parts will become stable and the line will become almost horizontal. Additionally, i and c both go down to zero. That is just the disease-free equilibrium.

\subsection{Parameters Settings with Endemic Equilibrium}

We may set the parameters as sown in Table 2. Also, $R_{0}=2.1653>1$ and $R_{1}=0.8087<1$.

However, as we mentioned in section 7 , we will provide different consequences under the existing model which we refer to here as the SIR model and the SIRC model.

The BRN for the SIR model is $R_{0}=\beta b /[\gamma(b+\sigma)]=0.1547<1$ (Xiao Y, 2002). According the definition of the BRN, under the given parameters settings, the SIR model will produce the disease-free result and the SIRC model will generate the endemic result, which are contradictory

According to the Theorem 5.2, the system has an endemic equilibrium with local asymptotic stability.

Table 2. Parameter Settings

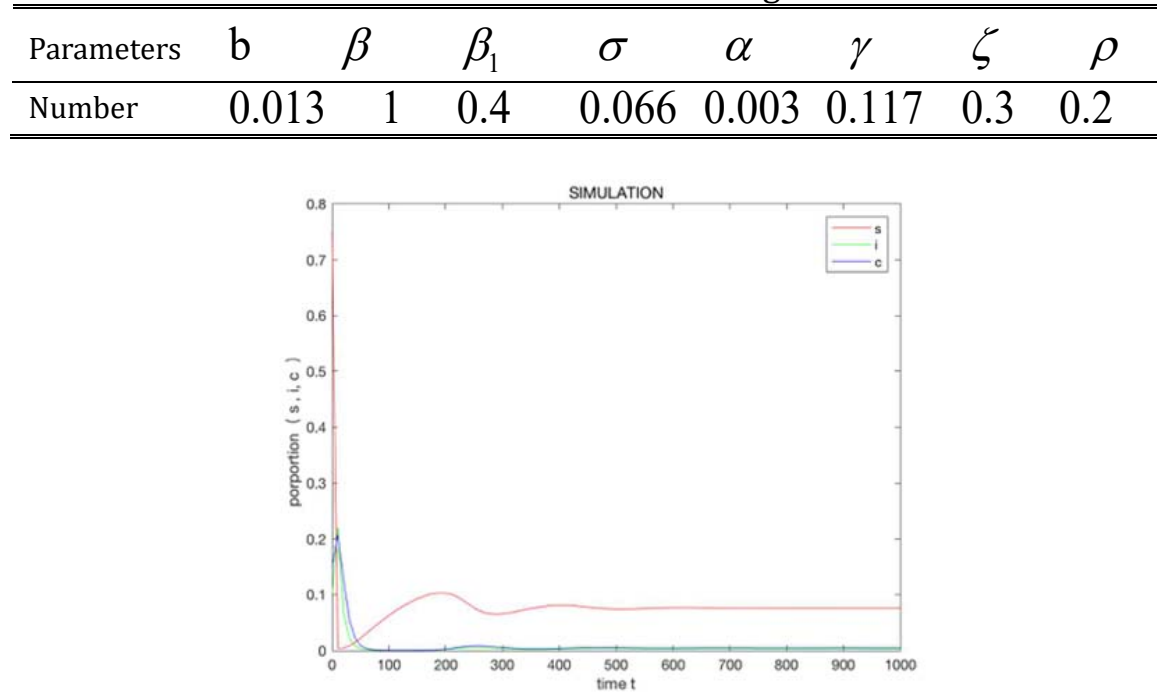

Fig. 3. Simulation with endemic equilibrium. 
The results presented in Fig. 3 reveal that, after chaos, the proportion of three parts will become stable and the line will become almost horizontal. All the three parts have a positive proportion. That is just the endemic equilibrium.

\section{References}

[1] Ma, Z. (2009). Dynamical modeling and analysis of epidemics. World Scientific.

[2] Hethcote, H. W. (2000). The mathematics of infectious diseases. SIAM Review, 42(4), 599-653.

[3] Coddington, E. A., \& Levinson, N. (1955). Theory of ordinary differential equations. Tata McGraw-Hill Education.

[4] Teschl, G. (2012). Ordinary differential equations and dynamical systems. American Mathematical Soc. (Vol. 140).

[5] Li, G., \& Jin, Z. (2005). Global stability of a SEIR epidemic model with infectious force in latent, infected and immune period. Chaos, Solitons \& Fractals, 25(5), 1177-1184.

[6] Graham, B. S. (2012). Dermatologic Manifestations of Hand-Foot--and-Mouth Disease.

[7] Roy, N, \& Halder, N. (2012). Compartmental modeling of hand, foot and mouth infectious disease (HFMD). Research Journal of Applied Sciences, 5(3), 177-182.

[8] Yang, J. Y., Chen, Y., \& Zhang, F. Q. (2013). Stability analysis and optimal control of a hand-foot-mouth disease (HFMD) model. Journal of Applied Mathematics and Computing, 41(1-2), 99-117.

[9] Roy, N. (2012). Mathematical modeling of hand-foot-mouth disease: quarantine as a control measure. International Journal of Advanced Scientific Engineering and Technological Research, 1(2).

[10] Zhu, Q., Hao, Y., Ma, J., Yu, S., \& Wang, Y. (2011). Surveillance of hand, foot, and mouth disease in mainland China (2008-2009). Biomedical and Environmental Sciences, 24(4), 349.

[11] Li, Y., Zhang, J., Zhang, X. (2014). Modelling and preventive measures of hand, foot and mouth disease (HFMD) in China. International Journal of Environmental Research \& Public Health, 11(3), 3108-17.

[12] Wang, M., Wenwen, M. A., \& Sun, M., et al. (2014). Research for transmission of hand-foot-mouth disease based on mathematical model. Journal of Biomedical Engineering Research.

[13] Ma, E., Wong, S., \& Wong, C., et al. (2011). Effects of public health interventions in reducing transmission of hand, foot, and mouth disease. Pediatric Infectious Disease Journal, 30(5), 432-435.

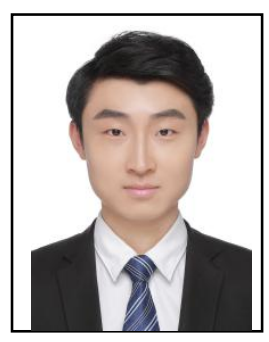

Ruzhang Zhao is a senior student of the Department of Mathematical Science, Tsinghua University. His research interests include ordinary differential equation and statistics.

Lijun Yang is the associate professor of the Department of Mathematical Science, Tsinghua University. His research interests include ordinary differential equation. 